\title{
Urolitíase no alotransplante renal ${ }^{1}$
}

\author{
Adauto José Cologna ${ }^{2}$, Antonio Carlos Pereira Martins ${ }^{3}$, Haylton Jorge Suaid ${ }^{4}$, Silvio Tucci Júnior ${ }^{2}$, Rodolfo Borges dos Reis ${ }^{5}$ \\ Ricardo Mesquita Paschoal ${ }^{6}$, André Luis Alonso Domingos ${ }^{6}$, Lucy Vieira da Silva-Lima ${ }^{7}$.
}

\begin{abstract}
Cologna AJ, Martins ACP, Suaid HJ, Tucci Jr S, Reis RB, Paschaol RM, Domingos ALA, Silva-Lima LV. Urolitíase no alotransplante renal. Acta Cir Bras (serial online) 2003 vol 18 suppl 5. Disponível em www.scielo.br/acb

RESUMO - Objetivo: A litíase urinária é uma complicação incomum no alotransplante renal, a incidência varia de 0,02 a 3,4\%. A maioria dos cálculos forma-se após o transplante, porém alguns podem ser transferidos junto com o enxerto para o hospedeiro. $\mathrm{O}$ tratamento desta complicação está baseado em alguns casos descritos na literatura. O objetivo deste trabalho é o de relatar a incidência da litíase renal no paciente com transplante renal, assim como a conduta adotada no HCFMRPUSP. Métodos: Foram analisados 953 pacientes submetidos a transplante renal no HCFMRPUSP, de fevereiro 1968 a maio de 2003. A idade média foi de 47,2 anos (35 a 63 anos). Em 09 pacientes, o rim foi proveniente de doador cadáver e apenas 01 doador vivo. Resultados: Foram diagnosticados 10 casos de litíase $(1,05 \%)$. Em 02 pacientes $(20 \%)$ o cálculo foi diagnosticado no intraoperatório, em 01 (10\%) no peri-operatório $\left(5^{\circ}\right.$. dia), os 07 restantes (70\%) no pós-operatório tardio. Em 04 pacientes (57\%) não havia sintomatologia específica, 02 (29\%) apresentaram ITU, em 03 (43\%) ocorreu elevação da creatinina sérica. De 8 pacientes com litíase no pós-operatorio, em 06 os cálculos estavam localizados no rim e 02 no ureter. Dos pacientes com cálculos renais, 02 foram observados, 02 submetidos a LECO, 01 a nefrolitripsia percutânea, 01 à pielolitotomia. Em 01 paciente com cálculo ureteral foi realizada pielovesicostomia (cálculo + estenose), no outro paciente foi feita a ureterorrenoscopia retrógrada. Conclusão: A urolitíase é complicação rara no transplante renal, a conduta terapêutica no pós-operatório tardio é semelhante à da população geral.
\end{abstract}

DESCRITORES: Litíase. Transplante renal.

\section{INTRODUÇÃO}

A Litíase urinária representa uma complicação urológica pouco comum no alotransplante renal, a incidência varia de 0,02 a 3,4\%1, 2,3,4,5,6. A maioria desses casos são considerados formações de novo, são cálculos que se formam no rim transplantado no pós-operatório tardio $6,8,9,10$. Não obstante, existem alguns relatos sugerindo que os cálculos são mais freqüentemente transferidos juntamente com os rins, do que se imaginava previamente ${ }^{8,11}$.

Pelas peculiaridades dos receptores renais, o quadro clínico da litíase renal geralmente é atípico. Em razão disso pode ocorrer retardo no diagnóstico ${ }^{4}$ ${ }^{11}$, além da possibilidade do quadro clínico ser confundido com episódios de rejeição aguda ${ }^{9}$.

Várias estratégias terapêuticas tem sido propostas para a resolução da litíase no enxerto renal. Entretanto, não há uma padronização de conduta a ser seguida, isto provavelmente, se deve ao número escasso de casos ${ }^{9,12,13,14 .}$

$\mathrm{O}$ objetivo deste trabalho é apresentar a experiência da Divisão de Urologia da Faculdade de Medicina de Ribeirão Preto da Universidade de São Paulo, com o diagnóstico e com a conduta a litíase urinária no alotransplante renal.

\section{MÉTODOS}

Foram analisados retrospectivamente os prontuários de 953 pacientes submetidos a alotransplante renal, no Hospital das Clínicas da Faculda- de de Medicina de Ribeirão Preto da Universidade de São Paulo, no período de fevereiro de 1968 a maio de 2003.

Para a realização do transplante renal foi empregada a técnica cirúrgica convencional, ou seja, o rim foi colocado em uma das fossas ilíacas, sendo os vasos renais anastomozados nos vasos ilíacos, o reimplante ureteral feito pela técnica de LichGregoire. A terapêutica imunossupressora foi variada. Para o diagnóstico da litíase foram realizados exame de urina (tipo I e cultura), dosagens de creatinina sérica, RX simples de abdome, US ou tomografia computadorizada sem contraste.

\section{RESULTADOS}

Dos 953 pacientes analisados, $10(1,05 \%)$ apresentaram litíase no rim transplantado (5 mulheres e 5 homens). A idade variou de 34 a 63 anos (média de 47,2 anos). Nove pacientes receberam rim de doador cadáver e apenas 1 de doador vivo relacionado.

Dois cálculos foram identificados no intraoperatório (20\%), ambos localizados no ureter; 1 foi diagnosticado no perioperatório $(10 \%)$ e alojado no cálice; nos 7 pacientes restantes os cálculos foram diagnosticados no período pós-operatório tardio (70\%), 3 caliciais, 2 piélicos e 2 ureterais. $\mathrm{O}$ intervalo entre o transplante e o diagnóstico da litíase, para o último grupo mencionado anteriormente, variou de 6 meses a 158 meses. O tamanho oscilou de 2 a $15 \mathrm{~mm}$ (média de 7,4 mm).
Em 4 dos 7 pacientes (57\%) cujo diagnóstico foi feito no pós-operatório tardio, não havia sintomatologia específica, 2 (29\%) apresentaram infecção urinária e, em 3 (43\%) ocorreu elevação da creatinina sérica. O diagnóstico da litíase calicial no $5^{\circ}$. dia de pós-operatório foi feito de forma incidental, após a realização de US para avaliação do enxerto renal.

A resolução dos cálculos diagnosticados no intraoperatório foi feito no mesmo ato cirúrgico (1 foi eliminado espontaneamente após o início da diurese, o outro foi removido após a secção do ureter distal). Dos 8 pacientes restantes; 2 ficaram em observação (ambos faleceram em decorrência de complicações não relacionadas com a litíase); 2 foram submetidos a pielolitotomia; 2 a LECO; 1 a nefrolitotripsia percutânea; 1 a ureterrenoscopia retrógrada. Todos os casos foram resolvidos sem a necessidade de terapêuticas complementares, em apenas 2 pacientes foi colocado duplo $\mathrm{J}$ (1 previamente a LECO e 1 após a ureterorrenoscopia). Não ocorreu qualquer intercorrência com os procedimentos adotados.

Durante o seguimento (média de 52 meses) ocorreu recidiva da litíase em 2 casos.

\section{DISCUSSÃO}

A incidência $(1,05 \%)$ de litíase urinária no alotransplante renal observado no presente trabalho é semelhante à apresentada em outras séries ${ }^{1,2,3,4,5,6}$.

1. Trabalho realizado na Unidade de Transplante Renal do HCFMRPUSP.

2. Professor Assistente Doutor, Departamento de Cirurgia e Anatomia FMRPUSP.

3. Professor Titular, Departamento de Cirurgia e Anatomia FMRPUSP.

4. Professor Associado, Departamento de Cirurgia e Anatomia FMRPUSP.

5. Médico Assistente da Divisão de Urologia HCFMRPUSP.

6. Médico Residente da Divisão de Urologia do HCFMRPUSP.

7. Pós-Graduanda do Departamento de Cirurgia e Anatomia do HCFMRPUSP. 
Mais da metade (57\%) dos pacientes com transplante renal e litíase não apresentaram nenhum tipo de sintomatologia dolorosa, portanto com quadro clínico atípico. A explicação plausível para este fato ocorre por conta da denervação do rim transplantado ${ }^{1,5,6,11}$. Percentual elevado de pacientes (43\%) cursaram com elevação dos níveis de creatinina sérica. Este evento deve ser analisado com cautela pois se associado a outros aspectos clínicos pode gerar confusão com episódio de rejeição aguda do enxerto ${ }^{9}$.

Os casos cujo diagnóstico da litíase foi feito no momento do transplante $(20 \%)$, aproveitou-se o mesmo ato cirúrgico para resolução dos mesmos. A presença de cálculo não complicado não constitui contra indicação para o prosseguimento do ato cirúrgico, no entanto quando há associação com infecção o transplante deve ser suspenso ${ }^{8}$.

Pelas peculiaridades do hospedeiro (cirurgia recente em fase de cicatrização, imunossupressão elevada, funcão renal limítrofe) o tratamento do cálculo diagnosticado no perioperatório constitui um verdadeiro desafio clínico, havendo relutância em indicar algum tipo de procedimento. Bhadauria e col. ${ }^{15}$, relataram 2 casos tratados com LECO, outros autores descrevem casos de nefrolitotripsia percutânea realizada de 1 a 2 meses após o transplante com mínima morbidade e conservação da função renal $^{11,13}$. Outra opção seria a observação cautelosa. No único caso relatado no presente trabalho optou-se pela observação inicial (4 meses) e posterior sessão de LECO, com sucesso.

Certamente, os cálculos diagnosticados no período perioperatório são transferidos acidentalmente para o receptor. Existem controvérsias com respeito a realização de exames de imagens para a detecção de litíase no rim doado. Para alguns, a realização de US transabdominal ou mesmo no intraoperatório, é factível, de baixo custo, pode ser feito paralelamente ao transplante sem aumentar o tempo de isquemia, permite o diagnóstico oferecendo a possibilidade da remoção do cálculo no mesmo ato cirúrgico, diminuindo a morbidade e futuros riscos ${ }^{8}$. Para outros, é difícil a avaliação real da relação custo/benefício pelo reduzido número de pacientes ${ }^{11}$. Nos casos relatados no presente trabalho não foi realizado US ou mesmo RX do doador.
A conduta adotada para os pacientes cujos cálculos foram diagnosticados no pós-operatório tardio $(70 \%)$, foi semelhante à conduta tomada para os pacientes portadores de litíase da população geral. Escolhendo-se um dos vários métodos terapêuticos disponíveis no arsena urológico moderno (LECO, nefrolitotripsia percutânea, ureterorrenoscopia), com as vantagens e desvantagens inerentes a cada um $^{15}$ 16. A cirurgia aberta deve ser evitada. Embora tenha sido utilizada em 02 casos foi justificada em um caso por ser a única opção no momento, no outro, o cálculo estava associado a estenose ureteral, havendo a necessidade de realização de uma derivação urinária (pielovesicostomia). Em alguns casos pode-se adotar a conduta da observação. Nesta circunstância o seguimento deve ser rigoroso, visto que uma hidronefrose silenciosa pode ocasionar a queda da função renal e, se ocorrer associação com infecção urinária, o quadro pode ser fatal ${ }^{17}$.

\section{CONCLUSÕES}

1 - A litíase urinária é uma complicação rara no transplante renal.

2 - A presença de um cálculo simples no momento da cirurgia, não contraindica o transplante.

3 - A conduta terapêutica para a litíase urinária diagnosticada no pós-operatório tardio é semelhante à aquelas adotadas para portadores de litíase da população geral. 4 - Na maioria dos pacientes o quadro clínico é atípico, cursando sem sintomatologia dolorosa.

\section{REFERÊNCIAS}

1. Benoit G, Blanchet $P$, Eschwege $P$. Occurrence and treatment of kidney graft lithiasis en a series of 1500 patients. Clin Transplant 1996; 10: 176-80.

2. Doehn C, Fornara P, Tiemer C. Renal transplant lithiasis. Transplantation Proccedings 2002; 34: 2222-3.

3. Harper M, Samuel CT, Hallson PC. Risk factors for calculus formation in patients with renal transplants. Br J Urol 1994; 74: 147-9.
4. Kim H, Cheigh LS, Ham HW. Urinary stones following renal transplantations. Korean S Int Med 2001; 16: 118-22.

5. Lancina-Martin JA, Garcia-Buitron JM, Diaz-Bermudez J. Urinary lithiasis in transplanted kidney. Arch Esp Urol 1997; 50: $141-50$.

6. Shoskes DA, Hanbury D, Cranston D. Urological complications in 1000 consecutive renal transplant recipients. J Urol 1995; 153: 18-21.

7. Torrecilla-Ortiz C, Gonzalez-Satue C, RieraCanals L. Incidence and treatment of urinary lithiasis in renal transplantation. Actas Urol Esp 2001; 25: 357-63.

8. Klinger HC, Kromer G, Lodde M. Urolithiasis en allograft kidneys. Urology 2002; 59: 344-8.

9. Rhee BK, Bretan Jr PN, Stoller ML. Urolithiasis en renal and combined pancreas/renal transplant recipients. J Urol 1999; 161: 1458-62.

10. Cho DK, Zackson DA, Cheigh J. Urinary calculi in renal transplant recipients. Transplantation 1998; 54: 889, 902.

11. Hsuech-Fu, LU, Shekarriz B, Stoller ML. Donorgifted allograft urolithiasis: early percutaneous management. Urology 2002; 59: 25-7.

12. Atala A, Steinbeck GS, Harty JL. Extrocorporeal shock wave lithotripsy in transplanted kidney. Urology 1993, 41: 60-2.

13. Citterio F, Grasseti F, Nanni G, et al. Accidental transplantation of a kidney with stones a case report. Transplant Proc 1991; 23: 2650 .

14. Minan-Cifuentes J, Arcia-Tapia E, Arcia de la Pena E. Percutaneous nephrolithotomy in transplanted kidney. Urology 1991; 38: 232-3.

15. Bhadauria RP, Ahlawat R, Kumar RV. Donor gifted allograft lithiasis: extracorporal shock wave lithotripsy with over table module using the Lithostar Plus. Urol Int 1995; 55: 51-5.

16. Caldwell TC, Burns JT. Current operative management of urinary calculi after renal transplantation, J Urol 1988; 111: 1006-9.

17. Van Gasbeke D, Zaiscman M, Simon S. Lithiasis complication of renal transplantation: the donor graft lithiasis concept. Urol Radiol 1985; 7: 157-60.

Cologna AJ, Martins ACP, Suaid HJ, Tucci Jr S, Reis RB, Paschaol RM, Domingos ALA, Silva-Lima LV. Urinary lithiasis in renal allografts. Acta Cir Bras [serial online] 2003 vol 18 suppl 5. Available in www.scielo.br/acb

ABSTRACT - Purpose: Urinary lithiasis is an uncommon complication in recipient of kidney allografts. The prevalence varies from 0.02 to $3.4 \%$. The majority of calculi arises de novo in the recipient, however some of them are transferred with the transplanted kidney. The treatment relies on few reports published previously. The aim of the study is to determine the prevalence of lithiasis as well as the treatment in an university hospital. Methods: We analyzed 953 recipients of renal transplant undertaken in Hospital das Clínicas - FMRP-USP, from February of 1968 to May of 2003. The mean age of patients bearing lithiasis was 47.2 years (range 35 to 63 years). Results: The prevalence of lithiasis was 10/953 (1.0\%). Nine patients received kidneys from cadaver donor and 1 from living donor. The diagnosis occurred during the surgery in $2(20 \%)$, within few days after transplantation in $1(10 \%)$ and in the late postoperative period in $7(70 \%)$. Seven patients had no complains, 2 had associated urinary tract infection and 1 a rise in serum creatinine. Of 8 cases with lithiasis in the postoperative period, the stones were localized in the kidney in 6 and in the ureter in 2 . Renal calculi were managed as follows: watchful-waiting -2 , extracorporeal lithotrypsy -2 , percutaneous nepholithotrypsy -1 and open pyelolithomy -1 . One patient with ureteric lithiasis associated ureteral stenosis underwent a pyelo-vesicostomy. The other patient with ureteric lithiasis was treated by retrograde endoscopic ureterolithothrypsy. Conclusion: Urinary lithiasis is rare in transplanted kidneys and can be managed as to the general population.

KEY WORDS: Renal allograft. Lithiasis.

Endereco para correspondência:

Adauto José Cologna

Departamento de Cirurgia e Anatomia FMRPUSP

Av. Bandeirantes, 3900, $9^{\circ}$ Andar

14.048-900 - Ribeirão Preto - SP

Email: ajcpçpgm@fmrp.sup.br 